\title{
Treatment outcome in patients with stage III breast cancer treated with neoadjuvant chemotherapy
}

\author{
RYUJI TAKAHASHI ${ }^{1}$, UHI TOH ${ }^{1}$, NOBUTAKA IWAKUMA ${ }^{1}$, MAI MISHIMA ${ }^{1}$, TERUHIKO FUJII ${ }^{1}$, \\ MIKI TAKENAKA ${ }^{1}$, KEIKO KOURA ${ }^{1}$, NAOKO SEKI ${ }^{2}$, AKIHIKO KAWAHARA ${ }^{3}$, \\ MASHAYOSHI KAGE ${ }^{3}$, ETSUYO OGO ${ }^{4}$ and KAZUO SHIROUZU $^{1}$ \\ ${ }^{1}$ Department of Surgery, ${ }^{2}$ Research Center for Innovative Cancer Therapy, Departments of ${ }^{3}$ Pathology and ${ }^{4}$ Radiology, \\ Kurume University School of Medicine, Kurume, Fukuoka 830-0011, Japan
}

Received April 25, 2013; Accepted August 22, 2013

DOI: $10.3892 /$ etm.2013.1289

\begin{abstract}
Despite the good responses of patients (pts) with stage III breast cancer to neoadjuvant chemotherapy (NAC), most eventually relapse and have a poor prognosis. We investigated the prognostic indicators in pts with stage III breast cancer treated with NAC, using epirubicin and/or docetaxel. A total of 22 women with stage III breast cancer underwent NAC between January 2005 and May 2011. The regimens of NAC comprised ED (epirubicin $60 \mathrm{mg} / \mathrm{m}^{2}$ and docetaxel $60 \mathrm{mg} / \mathrm{m}^{2}$ ) in 10 cases, FEC (fluorouracil $500 \mathrm{mg} / \mathrm{m}^{2}$, epirubicin 75-100 mg/ $\mathrm{m}^{2}$ and cyclophosphamide $500 \mathrm{mg} / \mathrm{m}^{2}$ ) in 10 cases and EC (epirubicin $60 \mathrm{mg} / \mathrm{m}^{2}$ and cyclophosphamide $600 \mathrm{mg} / \mathrm{m}^{2}$ ) in two cases. Following four cycles of each regimen, a further four cycles of D (docetaxel $70 \mathrm{mg} / \mathrm{m}^{2}$ ) were undertaken in nine cases. Subsequent to the completion of NAC and surgery, we assessed the clinicopathological results and performed prognostic analyses. Statistical analyses concerning disease-free survival (DFS) or overall survival (OS) were conducted by a Cox proportional hazard model. The median survival time was 66 months and there were 12 distant metastases and two local recurrences. Multivariate analyses showed the number of metastatic axillary lymph nodes (ALNs) [hazard ratio (HR), 1.079; $\mathrm{P}=0.023$ ] was correlated with $\mathrm{DFS}$, while the Ki-67 labeling index $(\mathrm{HR}, 1.109 ; \mathrm{P}=0.042)$ and the number of metastatic ALNs (HR, 1.087; $\mathrm{P}=0.023$ ) were correlated with OS. In conclusion, even if pts with stage III breast cancer show good responses to NAC using epirubicin and/or docetaxel, the majority eventually relapse and have a poor prognosis. The Ki-67 labeling index and the number of involved ALNs are suggested as prognostic indicators in stage III breast cancer.
\end{abstract}

Correspondence to: Dr Ryuji Takahashi, Department of Surgery, Kurume University School of Medicine, 67 Asahi-machi, Kurume, Fukuoka 830-0011, Japan

E-mail: takahashi_ryuuji@med.kurume-u.ac.jp

Key words: treatment outcome, prognostic indicator, neoadjuvant chemotherapy, stage III breast cancer

\section{Introduction}

Neoadjuvant chemotherapy (NAC) has become a standard therapy for patients (pts) with locally advanced breast cancer. Despite the initial good responses of pts with stage III breast cancer to NAC, these pts tend to relapse earlier and have worse prognoses than pts with stage I/II breast cancer. According to the databases of the American Cancer Society (1), the five-year overall survival (OS) rates for stage III breast cancer are $67 \%$ in stage IIIA and $41-49 \%$ in stage IIIB-IIIC. A number of prognostic factors for NAC have been correlated with OS and disease free survival (DFS) in locally advanced breast cancer, such as the triple-negative type, the human epidermal growth factor receptor 2 (HER2)-enriched type (hormone receptor negative/HER2 positive type) $(2,3)$, a pathological complete response (pCR) $(4,5)$ and the number of involved axillary lymph nodes (ALNs) at surgical staging $(6,7)$. However, only a small number of studies have investigated the prognostic indicators that are associated with long-term survival in pts with stage III breast cancer treated with NAC. The aim of this small-scale study was to investigate the prognostic indicators in pts with stage III breast cancer who have been treated with NAC.

\section{Patients and methods}

Study design and approval. This study was designed as an analysis of retrospective data in a single-facility, the Kurume University School of Medicine (Kurume, Japan). This observational study was approved by the Ethics Committee of Kurume University and all pts provided written informed consent for the treatment and publication of the data.

Eligibility criteria. Women under the age of 75 years with previously untreated clinical stage III breast cancer, who were diagnosed by mammography, ultrasonography, breast magnetic resonance imaging (MRI), core needle biopsy and positron emission tomography-computed tomography (PET/CT), were eligible for this study. Each pt had a locally advanced breast cancer with ALN involvement. The eligibility criteria also included adequate performance status [Eastern Cooperative Oncology Group (ECOG) performance 0-1], 
adequate hematology, renal and liver function and an ejection fraction $\geq 60 \%$, confirmed by ultrasonic cardiography. Pts who had an otherwise adverse medical history, another malignancy, contralateral breast cancer and/or a severe systemic condition were excluded.

NAC regimens and surgical methods. Three different NAC regimens were used: $\mathrm{ED}\left(60 \mathrm{mg} / \mathrm{m}^{2}\right.$ epirubicin and $60 \mathrm{mg} / \mathrm{m}^{2}$ docetaxel), FEC (500 mg/m² fluorouracil, $75-100 \mathrm{mg} / \mathrm{m}^{2}$ epirubicin, and $500 \mathrm{mg} / \mathrm{m}^{2}$ cyclophosphamide) and EC (60 mg/m epirubicin and $600 \mathrm{mg} / \mathrm{m}^{2}$ cyclophosphamide). For the pts who underwent EC and most of the pts who underwent FEC, a further four cycles of D (docetaxel $70 \mathrm{mg} / \mathrm{m}^{2}$ ) were then administered. Each chemotherapy regimen was administered every three weeks for four cycles; however, this interval was prolonged by at least one week if the pt did not recover from the adverse effects. Subsequent to the completion of the four cycles of NAC, we evaluated the clinical responses and performed surgery within 2-3 weeks. The surgical methods included Patey's procedure in three pts, mastectomy in $16 \mathrm{pts}$ and lumpectomy in three pts. All pts underwent level I+II ALN dissection. In addition, the three pts who received Patey's procedure underwent level III lymph node dissection.

Adjuvant therapy after surgery. Following surgery, extensional adjuvant chemotherapy was administered to $13 / 22$ pts (59\%) who had numerous ALN metastases ( $\geq 4$ positive nodes) and/or poor pathological responses to NAC. Each regimen of extensional chemotherapy was selected by the clinician. Nine of the 22 pts (41\%), who had a positive HER2 status, were treated with adjuvant trastuzumab (initially $8 \mathrm{mg} / \mathrm{kg}$, followed by $6 \mathrm{mg} / \mathrm{kg}$ ) for 12 months. Subsequent to the completion of adjuvant chemotherapy, whole breast irradiation of 50 Gy was performed for the pts who underwent a lumpectomy, while chest wall and regional lymph node irradiation of 50-60 Gy was performed for the majority of the pts. In addition, postmenopausal pts were treated with aromatase inhibitors for $\geq 5$ years, whereas premenopausal pts were given tamoxifen until menopause, prior to being switched to aromatase inhibitors.

Assessment of NAC in stage III breat cancer. To compare the efficacy of NAC using epirubicin and/or docetaxel in stage III breast cancer, we investigated 31 pts with stage III breast cancer who were treated with adjuvant chemotherapy between 1996 and 2005.

Evaluation of chemotherapy responses and toxicities. The clinical response was assessed based on a physical examination, mammography, ultrasonography, MRI and CT according to the Response Evaluation Criteria In Solid Tumors (RECIST) version 1.1 criteria (8). A clinically complete response (cCR) was defined as the disappearance of all known lesions; a clinically partial response (cPR) was defined as a $\geq 30 \%$ reduction in the sum of the longest diameter (LD) of the primary lesion; progressive disease (PD) was defined as a $\geq 20 \%$ increase in the sum of the LD of the primary lesion and stable disease (SD) was defined as neither sufficient shrinkage to qualify for CPR nor sufficient increase to qualify for PD. The efficacy of NAC was examined in the surgical specimens, while the Ki-67 labeling index was examined in the pre-treatment biopsy specimens. The pathological response was assessed based on the histological changes in the invasive area by the Japanese Breast Cancer Society criteria (9). A pCR was defined as no residual invasive cancer in the breast tissue, regardless of the ALN status, while the grade 0 response indicated no cancerous degeneration. A grade 2 response was defined as $\geq 2 / 3$ cancerous degeneration or a small amount of invasive cancer in the specimen, while a grade 1 response was defined as $<2 / 3$ cancerous degeneration in the specimen. The number of involved ALNs was confirmed in the dissected ALN specimen by the pathologist. In addition, toxicities of the NAC were graded by the ECOG common toxicity criteria.

Statistical analysis. OS and DFS were considered from the onset of NAC and from the day of breast surgery, respectively. The statistical analysis was conducted using JMP version 9.0 statistical software (SAS Institute, Inc., Cary, NC, USA). The correlation analysis was performed to compare two variables, including categorical and continuous variables. Univariate survival analyses to investigate predictive factors for OS and DFS were performed with a Cox proportional hazard model. The significant factors $(\mathrm{P}<0.05)$ were entered into a Cox multivariate regression model to analyze the potential simultaneous effects of the predictors of OS and DFS identified by univariate analyses. The survival analysis of the most significant factor was performed with the Kaplan-Meier method, and comparisons between the survival curves were performed with the log-rank test. Pt follow-up was performed in our hospital, from the beginning of chemotherapy either until mortality or the last visit of the pt.

\section{Results}

Pt characteristics. A total of 22 women with stage III breast cancer underwent NAC between January 2005 and May 2011. The median age was 55 years (range, 33-72 years) and the median follow-up period was 66 months (range, 9.3-90.0 months). The clinicopathological characteristics of the pts are shown in Table I. The observed intrinsic subtypes were as follows: six luminal, nine HER2-positive and seven triple-negative types. The pts' histological types showed invasive ductal carcinoma in 19 pts, mucinous carcinoma in two pts and invasive lobular carcinoma in one pt. There were no significant differences between the pts who underwent the FEC regimen and those who underwent a non-FEC regimen (ED, EC followed by D).

Clinical/pathological responses and toxicities. Clinical efficacy of NAC was observed in 64\% (cCR, 5\%; cPR, 59\%) of all pts (Table I). The remaining 36\% had SD (27\%) and PD (9\%). A younger age $(\mathrm{P}=0.010)$ and non-FEC regimen $(\mathrm{P}=0.005)$ were correlated with poor clinical responses. Pathological efficacy was observed in 36\% (pCR, 18\%; grade 2, 18\%) of all pts (Table I). The remaining 64\% showed grade $1(50 \%)$ and grade $0(14 \%)$ responses. There was no significant factor predicting whether the pathological response was likely to be good or poor.

Based on the ECOG common toxicity criteria, the most common toxicities were grade 1 and 2 neutropenia $(n=12$, Table II). One pt required an admission for grade 4 neutro- 
Table I. Characteristics of the patients treated with NAC.

\begin{tabular}{|c|c|}
\hline Characteristic & $\mathrm{n}(\%)^{\mathrm{a}}$ \\
\hline Median age [years (range)] & $55(33-72)$ \\
\hline \multicolumn{2}{|l|}{ Menopausal status } \\
\hline Premenopause & $9(41)$ \\
\hline Postmenopause & $13(59)$ \\
\hline \multicolumn{2}{|l|}{ Histological type } \\
\hline Invasive ductal carcinoma & $19(86)$ \\
\hline Mucinous carcinoma & $2(9)$ \\
\hline Invasive lobular carcinoma & $1(5)$ \\
\hline \multicolumn{2}{|l|}{ Nuclear grade } \\
\hline I-II & $14(64)$ \\
\hline III & $8(36)$ \\
\hline \multicolumn{2}{|l|}{ Intrinsic subtype } \\
\hline Luminal & $6(27)$ \\
\hline HER2-positive & $9(41)$ \\
\hline Triple negative & $7(32)$ \\
\hline \multicolumn{2}{|l|}{ NAC regimen } \\
\hline FEC (+D) & $10(45)$ \\
\hline ED & $10(45)$ \\
\hline $\mathrm{EC}+\mathrm{D}$ & $2(10)$ \\
\hline \multicolumn{2}{|l|}{ Initial tumor size (cm) } \\
\hline$\leq 2$ & $3(13)$ \\
\hline$>2, \leq 5$ & $9(41)$ \\
\hline$>5$ & $10(46)$ \\
\hline \multicolumn{2}{|l|}{ Initial axillary nodal status } \\
\hline $\mathrm{N} 1$ & $6(27)$ \\
\hline $\mathrm{N} 2$ & $11(50)$ \\
\hline N3 & $5(23)$ \\
\hline \multicolumn{2}{|l|}{ Clinical stage } \\
\hline Stage IIIA & $10(45)$ \\
\hline Stage IIIB & $7(32)$ \\
\hline Stage IIIC & $5(23)$ \\
\hline \multicolumn{2}{|l|}{ Surgical method } \\
\hline Lumpectomy & $3(13)$ \\
\hline Mastectomy & $16(73)$ \\
\hline Patey's procedure & $3(13)$ \\
\hline \multicolumn{2}{|l|}{ Radiotherapy } \\
\hline Yes & $16(73)$ \\
\hline No & $6(27)$ \\
\hline \multicolumn{2}{|l|}{ Clinical response } \\
\hline $\mathrm{CR} / \mathrm{PR}$ & $1 / 13(5 / 59)$ \\
\hline $\mathrm{SD} / \mathrm{PD}$ & $6 / 2(27 / 9)$ \\
\hline \multicolumn{2}{|l|}{ Pathological response } \\
\hline $\mathrm{pCR} /$ grade 2 & $4 / 4(18 / 18)$ \\
\hline grade $0 /$ grade 1 & $3 / 11(14 / 50)$ \\
\hline
\end{tabular}

NAC, neoadjuvant chemotherapy; FEC, 75-100 mg/m² epirubicin, $500 \mathrm{mg} / \mathrm{m}^{2}$ fluorouracil, $500 \mathrm{mg} / \mathrm{m}^{2}$ cyclophosphamide; D, docetaxel $70 \mathrm{mg} / \mathrm{m}^{2} ; \mathrm{ED}, 60 \mathrm{mg} / \mathrm{m}^{2}$ epirubicin, $60 \mathrm{mg} / \mathrm{m}^{2}$ docetaxel; EC, $60 \mathrm{mg} / \mathrm{m}^{2}$ epirubicin, $600 \mathrm{mg} / \mathrm{m}^{2}$ cyclophosphamide; CR, complete resonse; PR, partial response; $\mathrm{SD}$, stable disease; $\mathrm{PD}$, progressive disease; $\mathrm{pCR}$, pathological complete response. ${ }^{\mathrm{a}}$ For age, the range not $\%$ is shown.
Table II. Adverse events of NAC.

\begin{tabular}{|c|c|c|c|}
\hline Adverse event & Grade 1-2 & Grade 3 & Grade 4 \\
\hline \multicolumn{4}{|l|}{ Constitutinal symptom } \\
\hline Fever & 5 & 1 & \\
\hline Malaise & 2 & & \\
\hline \multicolumn{4}{|l|}{ Gastrointestinal } \\
\hline Anorexia & 7 & & \\
\hline Nausea & 4 & & \\
\hline Diarrhea & 3 & & \\
\hline Oral mucositis & 3 & & \\
\hline \multicolumn{4}{|l|}{ Neurological } \\
\hline Dysgeusia & 3 & & \\
\hline Stroke & 1 & & \\
\hline \multicolumn{4}{|l|}{ Blood/bone marrow } \\
\hline Anemia & 3 & 1 & \\
\hline Neutropenia & 12 & 6 & 1 \\
\hline Thrombocytopenia & & 1 & \\
\hline \multicolumn{4}{|l|}{ Laboratory } \\
\hline AST/ALT elevation & 2 & & \\
\hline
\end{tabular}

NAC, neoadjuvant chemotherapy; AST, aspartate aminotransferase; ALT, alanine aminotransferase.

penia, and six pts were classified as grade 3 . The other grade 3 toxicities are shown in Table II. Additional common toxicities were anorexia $(n=7)$ and fever $(n=6)$. The majority of the pts experienced alopecia.

Prognostic factors associated with OS and DFS. The median survival time was 66 months, and 11/22 pts (50\%) succumbed to refractory breast cancer. There were 12 distant metastases (five brain metastases, five lung metastases, one bone metastasis and one subclavian lymph nodes metastasis) and two local recurrences. pCR was not a prognostic factor for the success of NAC in this study. Univariate analyses showed that the triple-negative type, positive status of estrogen receptor and the number of involved ALNs were correlated with DFS, while the triple-negative type, Ki-67 labeling index (\%), pathological tumor size $(\mathrm{cm})$ and the number of involved ALNs were correlated with OS (Table III). Multivariate analyses showed that the number of involved ALNs [hazard ratio (HR), 1.079; 95\% confidence interval (CI), 1.011-1.155; $\mathrm{P}=0.023$ ] was correlated with DFS, while the Ki-67 labeling index (HR, 1.109; 95\% CI, 1.004-1.265; $\mathrm{P}=0.042)$ and the number of involved ALNs (HR, 1.087; 95\% CI, 1.012-1.180; $\mathrm{P}=0.023)$ were correlated with OS (Table III).

Treatment outcome of the pts with confirmed $p C R$. We assessed the outcome of four pts with observed pCR subsequent to surgery (Table IV). The five-year survival rate of these pts was $75 \%$ (3/4 pts) and 2/4 pts (50\%) suffered from a relapse of the breast cancer. These two pts relapsed with brain metastasis, having had a short DFS (2.9 and 7.9 months). For the four pts with observed pCR subsequent to surgery, initial staging, 
Table III. Uni- and multivariate analyses of the clinicopathological factors associated with overall and disease-free survival.

\begin{tabular}{|c|c|c|c|c|c|c|c|c|}
\hline \multirow[b]{3}{*}{ Factor } & \multicolumn{4}{|c|}{ Overall survival } & \multicolumn{4}{|c|}{ Disease-free survival } \\
\hline & \multicolumn{2}{|c|}{ Univariate } & \multicolumn{2}{|l|}{ Multivariate } & \multicolumn{2}{|c|}{ Univariate } & \multicolumn{2}{|l|}{ Multivariate } \\
\hline & HR & P-value & HR $(95 \%$ CI $)$ & P-value & HR & P-value & HR $(95 \%$ CI $)$ & P-value \\
\hline \multicolumn{9}{|l|}{ Menopausal status } \\
\hline Post-/premenopause & 0.400 & 0.135 & & & 0.466 & 0.161 & & \\
\hline \multicolumn{9}{|l|}{ NAC regimen } \\
\hline FEC/non-FEC regimen & 1.081 & 0.903 & & & 0.595 & 0.344 & & \\
\hline \multicolumn{9}{|l|}{ Initial stage } \\
\hline IIIB-IIIC/IIIA & 0.835 & 0.769 & & & 1.343 & 0.584 & & \\
\hline \multicolumn{9}{|l|}{ Nuclear grade } \\
\hline III/I-II & 1.591 & 0.450 & & & 1.412 & 0.528 & & \\
\hline \multicolumn{9}{|l|}{ Triple-negative type } \\
\hline Yes/no & 27.99 & $<0.001$ & $9.905(0.692-274.6)$ & 0.091 & 3.329 & 0.047 & $1.206(0.111-7.789)$ & 0.857 \\
\hline \multicolumn{9}{|l|}{ Estrogen receptor } \\
\hline Positive/negative & 0.336 & 0.123 & & & 0.167 & 0.006 & $0.273(0.037-1.388)$ & 0.118 \\
\hline \multicolumn{9}{|l|}{ HER2 status } \\
\hline Positive/negative & 0.366 & 0.113 & & & 1.310 & 0.615 & & \\
\hline Ki-67 labeling index $(\%)$ & 1.120 & $<0.001$ & $1.109(1.004-1.265)$ & 0.042 & 1.048 & 0.057 & & \\
\hline Pathological tumor size $(\mathrm{cm})$ & 1.605 & 0.006 & $1.242(0.895-1.826)$ & 0.194 & 1.350 & 0.057 & & \\
\hline Involved ALNs (number) & 1.061 & 0.037 & $1.087(1.012-1.180)$ & 0.023 & 1.068 & 0.027 & $1.079(1.011-1.155)$ & 0.023 \\
\hline $\begin{array}{l}\text { Pathological responses } \\
\text { pCR orade } 2 \text { /orade } 1 \text { orade } 0\end{array}$ & 0348 & 0139 & & & 0383 & 0.112 & & \\
\hline
\end{tabular}

FEC, 75-100 mg/m² epirubicin, $500 \mathrm{mg} / \mathrm{m}^{2}$ fluorouracil, $500 \mathrm{mg} / \mathrm{m}^{2}$ cyclophosphamide; HR, hazard ratio; CI, confidence interval; NAC, neoadjuvant chemotherapy; HER2, human epidermal growth factor receptor 2; ALNs, axillary lymph nodes; pCR, pathological complete response.

Table IV. Outcomes of the patients with confirmed pCR.

\begin{tabular}{lcccccccc}
\hline Case no. & Outcome & DFS (months) & Relapse site & Adjuvant chemotherapy & Stage & Involved ALNs & Subtype Ki-67 (\%) \\
\hline 1 & Alive & 34.0 & - & - & IIIB & 0 & Luminal $^{\mathrm{a}}$ & 14.7 \\
2 & Alive & 2.9 & Brain & Docetaxel, trastuzumab & IIIC & 0 & HER2 $^{\mathrm{b}}$ & 7.1 \\
3 & Dead & 7.9 & Brain & Tegafur & IIIC & 1 & $\mathrm{TN}^{\mathrm{c}}$ & 55.2 \\
4 & Alive & 8.4 & - & - & IIIA & 1 & $\mathrm{TN}^{\mathrm{c}}$ & 42.0 \\
\hline
\end{tabular}

${ }^{\mathrm{a} L u m i n a l}$ type; ${ }^{\mathrm{b}}$ human epidermal growth factor receptor 2 (HER2)-positive type; 'triple-negative type. pCR, pathological complete response; DFS, disease-free survival; ALNs, axillary lymph nodes.

intrinsic subtype and Ki-67 labeling index were suggested as prognostic indicators.

Feasibility of NAC in stage III breast cancer. Comparisons of the characteristics for the pts treated with NAC and those treated with adjuvant therapy (AT) are shown in Table V. The pts treated with AT included a higher proportion of stage IIIA disease and a smaller proportion of stage IIIC disease $(\mathrm{P}=0.040)$. The majority of the pts treated with AT underwent four cycles of anthracycline- and/or taxane-based regimens using doxorubicin, epirubicin and/or paclitaxel. DFS and OS curves for the
NAC and AT groups are shown in Fig. 1. In our hospital, epirubicin and/or docetaxel-based NAC was found not contribute to enhanced survival in stage III breast cancer.

Prognostic indicators in stage III breast cancer pts treated with $N A C$. We compared DFS and OS curves between pts with NIN $<4$ and pts with NIN $\geq 4$, and a greater number of NIN $(\geq 4)$ was significantly correlated with poor prognoses. Tausch et al (10) observed that an increased number of involved nodes (NIN) and an increased ratio of involved to removed nodes (LNR) were significantly correlated with worse DFS and OS in univariate 
Table V. Characteristics of the NAC and AT groups.

\begin{tabular}{lccc}
\hline & $\begin{array}{c}\text { NAC group } \\
(\mathrm{n}=22)\end{array}$ & $\begin{array}{c}\text { AT group } \\
(\mathrm{n}=31)\end{array}$ & P-value \\
Characteristic & $55(33-72)$ & $52(37-77)$ & 0.564 \\
Age [years (range) & & & 0.904 \\
Histological type & 19 & 27 & \\
Invasive ductal carcinoma & 2 & 2 & \\
Invasive lobular carcinoma & & & \\
Mucinous carcinoma & & 2 & \\
Metaplastic carcinoma & 6 & 14 & \\
Intrinsic subtype & 9 & 9 & \\
Luminal type & 7 & 8 & \\
HER2-positive type & & & 0.409 \\
Triple-negative type & 10 & 23 & \\
Initial stage & 7 & 7 & \\
Stage IIIA & 5 & 1 & \\
Stage IIIB & & & \\
Stage IIIC & 2 & 6 & \\
Events of recurrence & 12 & 21 & \\
Local recurrence & & & \\
Distant metastasis & & & \\
\hline
\end{tabular}

NAC, neoadjuvant chemotherapy; AT, adjuvant therapy; HER2, human epidermal growth factor receptor 2 .
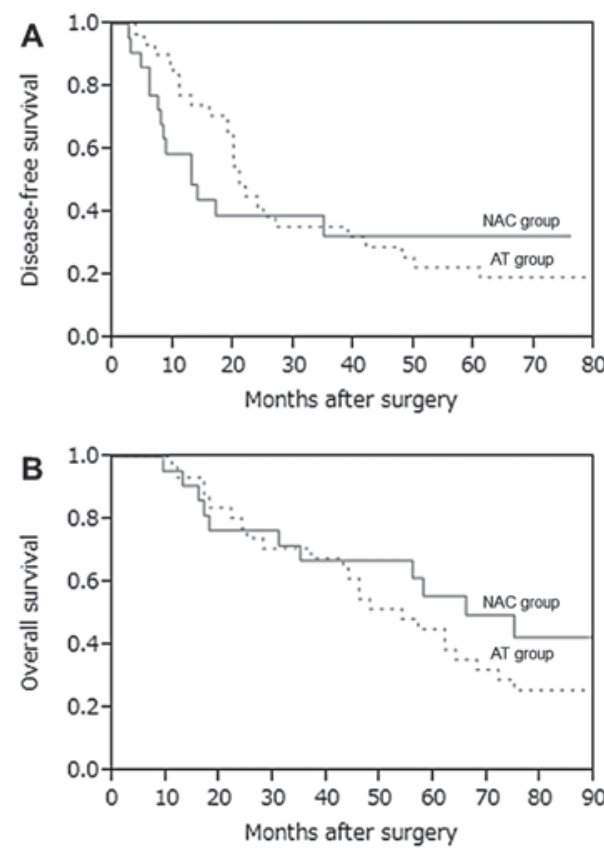

Figure 1. (A) Disease-free (DFS) and (B) overall survival (OS) curves for the neoadjuvant chemotherapy (NAC) and adjuvant therapy (AT) groups. No significant difference was identified between the two groups in the DFS and OS curves ( $\mathrm{P}=0.813$ and $\mathrm{P}=0.328$, respectively). The median survival time was 66 months in the NAC group and 54 months in the AT group.

and multivariate analyses $(\mathrm{P}<0.001)$. We compared DFS and OS curves between pts with NIN $<4$ and pts with NIN $\geq 4$ (Fig. 2). A high number of NINs $(\geq 4)$ was a significant prognostic
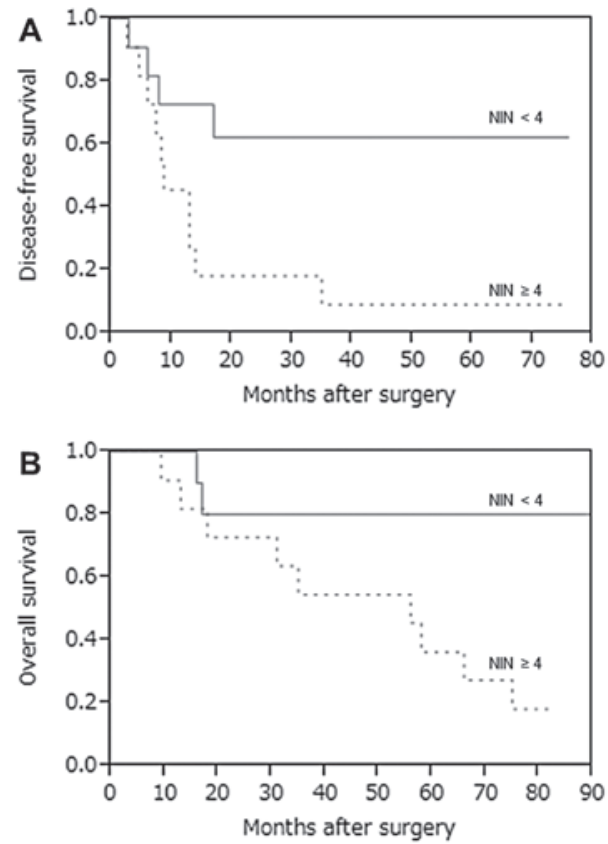

Figure 2. (A) Disease-free (DFS) and (B) overall survival (OS) curves for patients with different numbers of involved lymph nodes (NIN). A high NIN $(\geq 4)$ was a significant prognostic factor correlated with DFS and OS $(\mathrm{P}=0.025$ and $\mathrm{P}=0.024$, respectively).
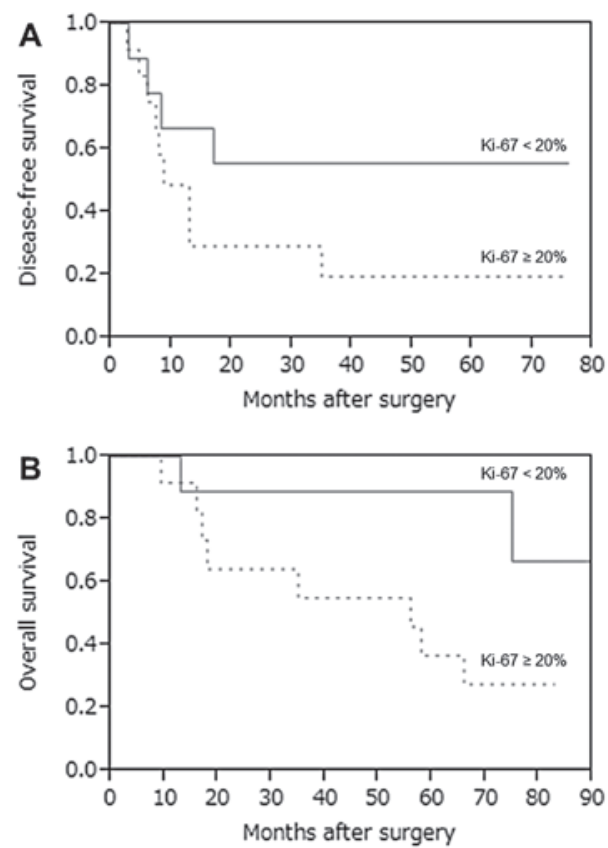

Figure 3. (A) Disease-free (DFS) and (B) overall survival (OS) curves according to the percentage of Ki-67. A high percentage of Ki-67 $(\geq 20 \%)$ appears to be as a prognostic factor correlated with OS $(\mathrm{P}=0.057)$, while no significant difference between low and high percentages of Ki-67 was observed in the DFS curves $(\mathrm{P}=0.183)$.

indicator correlated with DFS and OS $(\mathrm{P}=0.025$ and $\mathrm{P}=0.024$, respectively). In the current study, the $\mathrm{Ki}-67$ index was indicated to be as an independent prognostic factor for OS (HR, 1.109; 95\% CI, 1.004-1.265; P=0.042). However, achieving pCR subsequent to NAC was not associated with the Ki-67 labeling index $(\mathrm{P}=0.654$, Wilcoxon test $)$. Since the median percentage 
of Ki-67 was $21.3 \%$ (range, 7.1-55.2\%), we compared the DFS and OS curves with a cut-off value of Ki-67 at 20\% (Fig. 3). A high percentage of $\mathrm{Ki}-67(\geq 20 \%)$ was suggested as a prognostic indicator correlated with $\mathrm{OS}(\mathrm{P}=0.057)$, while there was no significant difference between the DFS curves $(\mathrm{P}=0.183)$.

\section{Discussion}

According to our five years of follow-up data for NAC in stage III breast cancer, the five-year OS and DFS rates were 50 and $36.4 \%$, respectively. The pCR and breast conserving rates after NAC were 18.2 and $13.6 \%$, respectively. Chávez-MacGregor and González-Angulo (4) suggested that achieving pCR after NAC correlated with improved DFS and OS and that, therefore, the amount of residual disease was a prognostic predictor. Ionta et al (11) analyzed 58/74 consecutive pts with stage IIIB breast cancer, who failed to achieve pCR following up to six cycles of a primary cisplatin, epirubicin and vinorelbine regimen. Following a median follow-up of 99 months, the 10-year DFS and OS rates were 37.6 and $50.3 \%$, respectively, which were significantly worse than those in the $\mathrm{pCR}$ group $(\mathrm{n}=16 ; \mathrm{P}=0.003$ and $\mathrm{P}=0.008$, respectively). Their results suggested that the number of residual ALNs and being negative for hormone receptors were strong predictors of poor outcomes, while the triple-negative type showed a trend towards early recurrence and mortality. Our results also suggested that the pathological tumor size subsequent to NAC and a triple-negative type were prognostic predictors. However, no significant difference was observed in the multivariate analysis. It was not possible to evaluate these predictors accurately; therefore, larger numbers of pts with stage III breast cancer treated with NAC are required for analysis.

With regard to the validity of NAC in stage III breast cancer, Tanioka et al (12) investigated the predictive factors of recurrence in 88 pts achieving pCR following NAC. During a median 46-month follow-up period, there were 12 recurrences, including eight distant metastases. Multivariate analyses showed that ALN metastasis (HR, 13.6; $\mathrm{P}<0.001)$ and HER2-positive type (HR, 5.0; $\mathrm{P}=0.019)$ were significant predictors of recurrence. In the current study, we observed two pts who experienced relapses of breast cancer into the brain out of the four pts who achieved pCR after NAC (Table IV). Although these two pts had a small number of involved ALNs, they were stage IIIC; one pt had a HER2-positive type and the other had a high percentage of Ki-67 (55.2\%). Yuan et al (13) revealed that NAC exhibited better recurrence control and DFS and OS rates than adjuvant chemotherapy in stage III breast cancer; however, it did not result in greater survival in stage II disease. By contrast, our results indicated that NAC may have no survival advantages compared with adjuvant chemotherapy in stage III breast cancer pts. Notably, higher frequencies of the triple-negative type and stage IIIB-IIIC breast cancer were shown in the NAC group. However, these results indicate the need to consider more tailored and effective NAC regimens for pts with stage III breast cancer.

Our results suggested that the Ki-67 labeling index and the number of involved ALNs are prognostic predictors in stage III breast cancer. In a recent study, Zhang et al (7) investigated axillary nodal staging in stage II/III breast cancer after NAC. The authors observed that the ypN staging adjusted by $\mathrm{pCR}$ following NAC may predict differential DFS. We showed that the ypN staging after NAC may be a prognostic indicator among stage III breast cancer pts, although the number of pts was too small to confirm this. A greater number of NIN $(\geq 4)$ after NAC may also be a predictive factor for recurrence or poor prognosis in our study. Furthermore, we evaluated the Ki-67 in pre-treatment biopsy specimens, due to the fact that tissue degeneration following chemotherapy often makes it difficult to identify Ki-67-positive tumor cells. In a recent review of Ki-67 data (14), Ki-67 was an independent prognostic factor for DFS (HR, 1.05-1.72) in multivariate analyses in seven randomized trials (level of evidence, I-B) and for OS (HR, 1.11-1.83) in univariate analyses in five trials. In addition, a high Ki-67 was observed to be correlated with immediate pCR with neoadjuvant therapy (level of evidence, II-B). I-B and II-B levels of evidence may be defined as follows: The I-B level of evidence applies to instances where a randomized controlled trial (RCT) was not specifically performed to assess the utility of the biomarker. The samples were stored during the study and analyzed when the study had finished, following a protocol. Only one validation study, or several studies with inconsistent results were desirable. For a II-B level of evidence, an RCT was not specifically performed to assess the utility of the biomarker. The samples were stored during the study and analyzed once the study had finished, following a protocol. One or more validation studies with consistent results were desirable. In the present study, the Ki-67 was indicated to be an independent prognostic factor for OS, and a high percentage of Ki-67 ( $\geq 20 \%)$ was correlated with poor prognosis, although there was no significant difference between the DFS curves. These results indicate that the $\mathrm{Ki}-67$ index in pre-treatment tumor tissues may be used as a prognostic indicator for localized advanced breast cancer pts.

In conclusion, even if pts with stage III breast cancer show good responses to NAC using anthracycline and/or taxanes, most eventually relapse and have a poor prognosis. The Ki-67 labeling index and the number of involved ALNs may be prognostic indicators in stage III breast cancer.

\section{Acknowledgements}

The authors thank Dr Tatsuyuki Kakuma (Biostatistic Center, Kurume University, Kurume, Japan) for supporting the data analysis in this study.

\section{References}

1. American Cancer Society: Breast cancer survival rates by stage. http://www.cancer.org/cancer/breastcancer/detailedguide/ breast-cancer-survival-by-stage. Accessed September 6, 2012.

2. Bhargava R, Beriwal S, Dabbs DJ, Ozbek U, Soran A Johnson RR, et al: Immunohistochemical surrogate markers of breast cancer molecular classes predicts response to neoadjuvant chemotherapy: a single institutional experience with 359 cases. Cancer 116: 1431-1439, 2010.

3. Carey LA, Dees EC, Sawyer L, Gatti L, Moore DT, Chollichio F, et al: The triple negative paradox: primary tumor chemosensitivity of breast cancer subtypes. Clin Cancer Res 13: 2329-2334, 2007.

4. Chávez-MacGregor M and González-Angulo AM: Breast cancer, neoadjuvant chemotherapy and residual disease. Clin Transl Oncol 12: 461-467, 2010.

5. Kong X, Moran MS, Zhang N, Haffy B and Yang Q: Metaanalysis confirms achieving pathological complete response after neoadjuvant chemotherapy predicts favourable prognosis for breast cancer patients. Eur J Cancer 47: 2084-2090, 2011. 
6. Zhang GC, Zhang YF, Xu FP, Qian XK, Guo ZB, Ren CY and Yao M: Axillary lymph node status, adjusted for pathologic complete response in breast and axilla after neoadjuvant chemotherapy, predicts differential disease-free survival in breast cancer. Curr Oncol 20: e180-192, 2013.

7. Kim J,Lee J, Chang E, Suh K, Lee C, Jee J and Shin H: Prognostic factors in patients with stage II/III breast cancer treated with adjuvant extension of neoadjuvant chemotherapy: a retrospective cohort study with ten-years of follow-up data. J Breast Cancer 14: 39-45, 2011.

8. Eisenhauer EA, Therasse P, Bogaerts J, Schwartz LH, Sargent $\mathrm{D}$, Ford R, et al: New response evaluation criteria in solid tumours: revised RECIST guideline (version 1.1). Eur J Cancer 45: 228-247, 2009.

9. Kurosumi M, Akashi-Tanaka S, Akiyama F, Komoike Y Mukai H, Nakamura S, et al; Committee for Production of Histopathological Criteria for Assessment of Therapeutic Response of Japanese Breast Cancer Society: Histopathological criteria for assessment of therapeutic response in breast cancer (2007 version). Breast Cancer 15: 5-7, 2008.

10. Tausch C, Taucher S, Dubsky P, Seifert M, Reitsamer R, Kwasny W, et al: Prognostic value of number of removed lymph nodes, number of involved lymph nodes, and lymph node ratio in 7502 breast cancer patients enrolled onto trials of the Austrian Breast and Colorectal Cancer Study Group (ABCSG). Ann Surg Oncol 19: 1808-1817, 2012.
11. Ionta MT, Atzori F, Deidda MC, Pusceddu V, Palmeri S, Frau B, et al: Long-term outcomes in stage IIIB breast cancer patients who achieved less than a pathological complete responses $(<\mathrm{pCR})$ after primary chemotherapy. Oncologist 14: 1051-1060, 2009.

12. Tanioka M, Shimizu C, Yonemori K, Yoshimura K, Tamura K, Kouno T, et al: Predictors of recurrence in breast cancer patients with a pathologic complete response after neoadjuvant chemotherapy. Br J Cancer 103: 297-302, 2010.

13. Yuan Z, Qu X, Zhang ZT and Wang Y: Neoadjuvant chemotherapy in patients with stage II and III breast cancer. Chin Med J (Engl) 122: 2993-2997, 2009.

14. Luporsi E, André F, Spyratos F, Martin PM, Jacqueimer J, Penault-Llorca F, et al: Ki-67: level of evidence and methodological considerations for its role in the clinical management of breast cancer: analytical and critical review. Breast Cancer Res Treat 132: 895-915, 2012. 